\title{
InSAR elevation bias caused by penetration into uniform volumes
}

\author{
Dall, Jørgen
}

Published in:

I E E E Transactions on Geoscience and Remote Sensing

Link to article, DOI:

10.1109/TGRS.2007.896613

Publication date:

2007

Document Version

Publisher's PDF, also known as Version of record

Link back to DTU Orbit

\section{Citation (APA):}

Dall, J. (2007). InSAR elevation bias caused by penetration into uniform volumes. I E E E Transactions on Geoscience and Remote Sensing, 45(7), 2319-2324. https://doi.org/10.1109/TGRS.2007.896613

\section{General rights}

Copyright and moral rights for the publications made accessible in the public portal are retained by the authors and/or other copyright owners and it is a condition of accessing publications that users recognise and abide by the legal requirements associated with these rights.

- Users may download and print one copy of any publication from the public portal for the purpose of private study or research.

- You may not further distribute the material or use it for any profit-making activity or commercial gain

- You may freely distribute the URL identifying the publication in the public portal

If you believe that this document breaches copyright please contact us providing details, and we will remove access to the work immediately and investigate your claim. 


\title{
InSAR Elevation Bias Caused by Penetration Into Uniform Volumes
}

\author{
Jørgen Dall, Member, IEEE
}

\begin{abstract}
Natural media like cold-land ice, vegetation, and dry sand are subject to a substantial penetration at microwave frequencies. For such media, the synthetic aperture radar (SAR) phase center is located below the surface, and consequently, the surface elevation determined with SAR interferometry (InSAR) is biased downward. For infinitely deep uniform volumes, the elevation bias is often equated with the penetration depth, but in this paper, it is shown that the two quantities generally differ. The interferometric bias is approximately equal to the two-way power-penetration depth if the latter is small compared to the ambiguity height, but for increasing penetration depth, the bias approaches one quarter of the ambiguity height. Consequently, no phase wrapping results even if the penetration depth exceeds the ambiguity height. The ratio of the InSAR elevation bias to the ambiguity height depends only on the ratio of the penetration depth to the ambiguity height, and the bias can be expressed in terms of the InSAR coherence magnitude, which makes it possible to correct the InSAR surface elevation for the bias. The volume depth can be considered infinite if it exceeds the penetration depth by a factor of two to five and if the surface scattering from the top and the bottom of the volume is negligible.
\end{abstract}

Index Terms-Elevation bias, interferometry, penetration, synthetic aperture radar (SAR), volume scattering.

\section{INTRODUCTION}

$\mathbf{S}$ YNTHETIC aperture radar (SAR) tomography [1] offers a direct measurement of the 3-D location of volume scatterers and their radar brightness. Single-baseline SAR interferometry (InSAR), on the other hand, relies on the assumption that the scatterers are located on a surface, and that, this surface has an unambiguous intersection with the circles that are defined by the range and Doppler measurements of the SAR (no layover). Volume scattering reduces the InSAR coherence, adds noise to the interferometric phase, and displaces the phase center from the surface of the volume. This displacement, in turn, is equivalent to a negative bias of the surface elevation that is determined directly from the InSAR data. However, in combination with a coherent scattering model, the InSAR technique can be adequately applied to certain types of volume scatterers. For instance, in polarimetric InSAR (Pol-InSAR) [2], [3], such scattering models are complementing the InSAR data, thereby overcoming the limitations inherent in the InSAR technique and enabling a vegetation volume and its underlying ground surface to be mapped [4].

Manuscript received May 26, 2006; revised February 20, 2007. This work was supported in part by the Danish Technical Research Council.

The author is with the Danish National Space Center, Technical University of Denmark, 2800 Kongens Lyngby, Denmark (e-mail: jd@space.dtu.dk).

Digital Object Identifier 10.1109/TGRS.2007.896613
Mapping the surface of an infinitely deep uniform volume is simpler than mapping a vegetated ground, but the penetration of the radar signal into the volume violates the basic InSAR assumption, even if the geometry is completely defined by a surface. Consequently, a coherent scattering model is also needed in this case.

The power-penetration depth and the elevation bias may be quite large for some types of volume scatterers. At C-band, about $9 \mathrm{~m}$ of penetration has been measured for dry cold firn [5]. In the percolation zone of the Greenland ice sheet, C-band penetration depths exceeding $11 \mathrm{~m}$ have been measured [6]. At L-band, the penetration depth is found to be 5-10 m larger than at C-band [5], but for smooth cold exposed ice, penetration depths up to 60-120 $\mathrm{m}$ have been observed [5].

A vegetation layer also exhibits a pronounced penetration effect, and if sufficiently thick and dense, it may seem to be infinitely thick. Forests are very diverse. For tropical rain forests, C-band InSAR data acquired during the Indonesian Radar Experiment campaign in 1996 (INDREX 1996) suggest a one-way extinction coefficient in the range of $0.15-0.3 \mathrm{~m}^{-1}$ [7], corresponding to a penetration length of 3-7 m, i.e., substantially less than the forest height. Topographic SAR (TOPSAR) results indicate an extinction coefficient of about $1 \mathrm{~dB} / \mathrm{m}$ [8], corresponding to a penetration length of $4 \mathrm{~m}$. For boreal coniferous forests, typical extinctions are $0.2-0.4 \mathrm{~dB} / \mathrm{m}$, corresponding to a penetration length of 11-22 $\mathrm{m}$ [8].

Yet, another medium subject to penetration is dry sand [9]. Images from the L-band Shuttle Imaging Radar mission (SIR-A) have revealed river channels beneath the sand of the Sahara desert, and a power-penetration length of at least $2.5 \mathrm{~m}$ ( $5 \mathrm{~m}$ one-way field penetration) has been calculated based on laboratory measurements of sand from the site in question [10].

The aforementioned ice "penetration depths" are actually elevation biases, since they are found either as the difference between calibrated InSAR and laser scanner digital elevation models (DEMs) [5], [6] or as the elevation difference between reflectors deployed on the ice surface and the surrounding ice, both are determined from an InSAR DEM [6].

The relationship between penetration and bias is the subject of this paper, whereas the relationship between various penetration and extinction parameters is straightforward: For a volume with exponential extinction, the penetration length $l$ is the oneway distance over which the power level decreases by a factor of $e$, which is the base of the natural logarithm. This penetration length is related to the one-way power-penetration depth

$$
d_{1}=l \cos \theta_{v}
$$


where $\theta_{v}$ is the refraction angle (in the volume). In addition, the penetration length is related to the one-way power-extinction coefficient

$$
\sigma=l^{-1}
$$

which corresponds to a power-extinction of $10 \sigma \log _{10}(e) \mathrm{dB} / \mathrm{m}$. The two-way extinction coefficient is a factor of two larger than the corresponding one-way coefficient, while the two-way penetration parameters are a factor of two smaller than the oneway penetration parameters.

This paper concerns the surface elevation bias resulting from penetration into a volume scatterer. This bias is defined as the difference between the InSAR elevation and the true surface elevation. The volume is assumed to be infinitely deep, and surface scattering is disregarded. For this scenario, the penetration depth has previously been related to the InSAR coherence [11], and for a corresponding finite-depth geometry, i.e., a volumeover-ground scenario, estimation of the ground elevation and volume thickness has been addressed intensively [4], [8], [12]. However, until now, the bias of the measured surface elevation has not been studied directly.

\section{COMPLEX InSAR COHERENCE}

An infinitely deep volume scatterer with a flat horizontal surface defines the geometry of interest. A Cartesian coordinate system is defined such that the surface is located at $z=0$, i.e., the extent of the scatterer is confined by $z<0$, and the extent of the air is confined by $z>0$. Initially though, a volume with a finite depth $D$ is considered in order to allow this paper to be based on existing work and to enable an analysis of the minimum depth required for the results to be applicable to the finite-depth volumes encountered in practice. Thus, to begin with, the volume is confined by $-D<z<0$.

The volume is characterized by an exponential extinction. The extinction coefficient accounts for the combined effect of absorption and scattering, but the relative contribution of the two mechanisms is irrelevant.

The volume is assumed to be uniform. This implies that the scattering coefficient per unit volume and the extinction coefficient do not have any spatial variation. These parameters may depend on the frequency and the polarization, but for the simple model in question, it is not necessary to exploit any such dependence in order to estimate and correct for the surface elevation bias. This means that the results are valid for both random volumes and oriented volumes.

The complex InSAR coherence (after "phase flattening" and "wavenumber shift filtering" [13]) for the defined volume has been previously derived [4], [8], [12]

$$
\gamma=\frac{\int_{-D}^{0} \exp \left(\left(d_{2}^{-1}+j k_{z}\right) z\right) d z}{\int_{-D}^{0} \exp \left(d_{2}^{-1} z\right) d z}
$$

where the effective vertical interferometric wavenumber in the volume is the derivative of the interferometric phase $\phi$ in the vertical direction

$$
k_{z}=\frac{\partial \phi}{\partial z} .
$$

Equation (3) is obtained by rewriting [4, eq. (14)] in terms of the two-way penetration depth $d_{2}$

$$
d_{2}=\frac{\cos \theta_{v}}{2 \sigma}
$$

and normalizing by the (constant) interferometric phase at the top of the volume $\phi_{s}$ rather than that at the bottom of the volume, i.e., $\angle \gamma=\phi-\phi_{s}$. The vertical wavenumber can be expressed in terms of the ambiguity height in the volume $h_{a}$, which is the elevation difference corresponding to a $2 \pi$ difference of the interferometric phase

$$
k_{z}=\frac{2 \pi}{h_{a}} .
$$

Using the result derived in the Appendix, the ambiguity height, in turn, can be computed as

$$
h_{a}=\frac{\lambda H \tan \theta_{a}}{p B_{\perp}} \sqrt{\frac{n^{2}-\sin ^{2} \theta_{a}}{n^{2} \cos \theta_{a}}}
$$

where $\lambda$ is the wavelength in air, $H$ is the altitude of the SAR with respect to the volume surface, $\theta_{a}$ is the incidence angle (in the air), $B_{\perp}$ is the component of the baseline perpendicular to the line-of-sight direction, $n$ is the refractive index, and $p$ equals two in ping-pong mode and otherwise one. In pingpong mode, each InSAR antenna transmits and receives its own signal as in repeat-pass interferometry. The $h_{a}$ given in (7) is an approximation, assuming that the penetration depth is much smaller than the total slant range. The last fraction is due to the refraction and does not appear in the expression for the ambiguity height in air [14].

The ambiguity height is positive when the interferometric phase is an increasing function of the target height, cf. (4), (6), and (7), and in this case also, the perpendicular baseline is defined as positive, cf. (7).

The volume integrals in (3) can easily be solved

$$
\gamma=\frac{d_{2}^{-1}}{\left(d_{2}^{-1}+j k_{z}\right)} \frac{1-\exp \left(-\left(d_{2}^{-1}+j k_{z}\right) D\right)}{1-\exp \left(-d_{2}^{-1} D\right)} .
$$

This coherence is consistent with the vegetation coherence of the interferometric water cloud model (IWCM) [15]. For an infinitely deep volume, i.e., for $D \rightarrow \infty$, the coherence is

$$
\gamma=\frac{1}{1+j 2 \pi d_{2} / h_{a}} .
$$

This coherence can be interpreted geometrically by rewriting it as [16]

$$
\gamma=\frac{1}{2}+\frac{1}{2} \frac{1-j 2 \pi d_{2} / h_{a}}{1+j 2 \pi d_{2} / h_{a}} .
$$

Here, the last fraction has a unity magnitude and a phase increasing from $0^{\circ}$ to $180^{\circ}$ when $d_{2}$ increases from zero to 


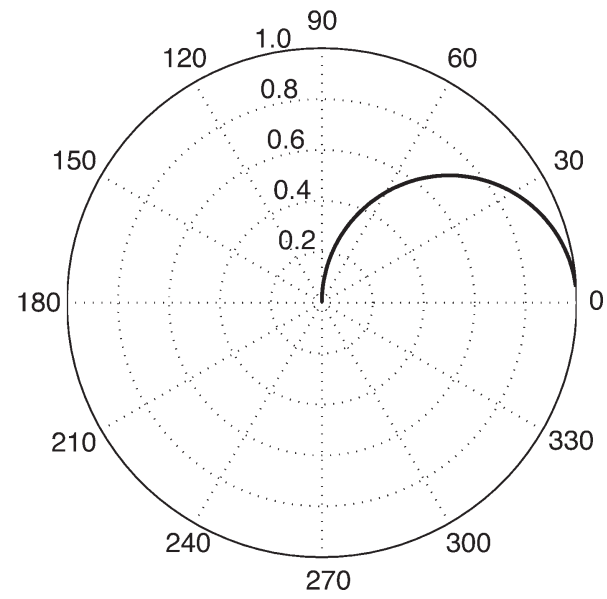

Fig. 1. InSAR coherence for an infinitely deep volume plotted in the complex plane for variable penetration to ambiguity ratio. The coherence is plotted for negative ambiguity height, and it is normalized by the interferometric phase of the volume surface such that the coherence is $e^{j 0}$ for zero penetration and $2^{-1 / 2} e^{j \pi / 4}$ when the two-way penetration depth equals the ambiguity height divided by $2 \pi$.

infinity and $h_{a}$ is negative (otherwise, from $0^{\circ}$ to $-180^{\circ}$ ). Hence, in the complex plane, (10) defines a semicircle with radius $1 / 2$ and center at $1 / 2$, as shown in Fig. 1 .

\section{INSAR ELEVATION BIAS}

The elevation bias is defined as the difference between the elevation measured directly with InSAR $h_{m}$ and the true surface elevation $h_{s}$

$$
\Delta_{h}=h_{m}-h_{s}
$$

With this definition, the bias is negative. In the following equations, the sign is explicitly taken into account, but elsewhere, it is often implicit. Due to the phase normalization of the coherence, the bias defined by (11) is proportional to the coherence phase

$$
\Delta_{h}=\angle \gamma / k_{z}=\angle \gamma h_{a} /(2 \pi) .
$$

It follows from (9) and (12) that the relative bias $\Delta_{h} / h_{a}$ only depends on the relative penetration depth $d_{2} / h_{a}$, and the dependence is plotted in Fig. 2.

According to (9), $\angle \gamma \approx-2 \pi d_{2} / h_{a}$ for small relative penetration depths, and hence

$$
\Delta_{h} \approx-d_{2} \quad \text { for } \quad d_{2} /\left|h_{a}\right| \ll 1 .
$$

Fig. 1 and (9) also show that $\angle \gamma \approx-\operatorname{sgn}\left(h_{a}\right) \pi / 2$ for large relative penetration depths, where sgn is the signum function. Consequently

$$
\Delta_{h} \approx-h_{a} / 4 \text { for } d_{2} /\left|h_{a}\right| \gg 1
$$

as shown in Fig. 2, where the relative bias approaches $-1 / 4$ for large relative penetration depths. Fig. 2 also confirms (13), as the $\Delta_{h} / h_{a}$ curve coincides with the $-d_{2} / h_{a}$ curve for small relative penetration depths. However, the curves diverge, and the InSAR bias is substantially smaller than the penetration

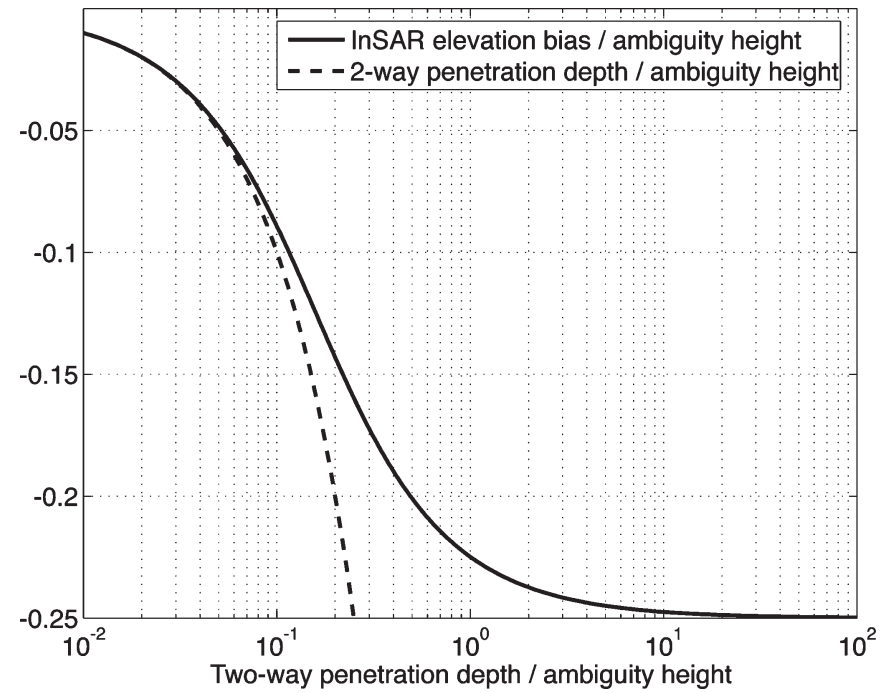

Fig. 2. Relative bias approaches the two-way penetration depth for small relative penetrations and one quarter of the ambiguity height for large relative penetration depths.

depth if the latter exceeds some $10 \%$ of the ambiguity height. It can be concluded as follows.

- The InSAR elevation bias is approximately equal to the two-way power-penetration depth if the latter is small compared with the ambiguity height.

- The InSAR elevation bias is approximately one quarter of the ambiguity height if the two-way power-penetration depth is large compared with the ambiguity height.

If the interferometric phase had been proportional to the penetration depth over the whole range of the penetration depths, the bias would wrap around for penetration depths exceeding the ambiguity height.

- The bias is not subject to any ambiguity resulting from phase wrapping, since the penetration cannot change the phase by more than $\pi / 2$.

This can be understood by considering the variation of the InSAR phasors with depth. In the layer defined by $-h_{a}<$ $z<0$, the phasor angles are evenly distributed over 0 to $2 \pi$, but due to the extinction, the uppermost phasors contribute the most, and the phasor sum ends up with an angle between 0 and $\pi / 2$ (for negative $k_{z}$ ). The smaller the extinction, the larger the resulting phasor angle, but the smaller the coherence, as the phasors with almost the same lengths add up to a small resulting phasor. The same applies to all other layers defined by $(k-1) h_{a}<z<k h_{a}$, where $k$ is a negative integer.

Even if the bias itself does not wrap, the biased phase could still wrap due to spatial gradients in either the mean surface height or the penetration depth, and the phase unwrapping may be impaired by the low coherence that accompanies a large bias.

\section{Bias Mitigation}

According to (11), the true surface elevation can be computed by estimating the bias and subtracting it from the elevation that is measured directly.

In practice, the phase of the coherence, as defined in (9) and plotted in Fig. 2, cannot be estimated directly, because the 


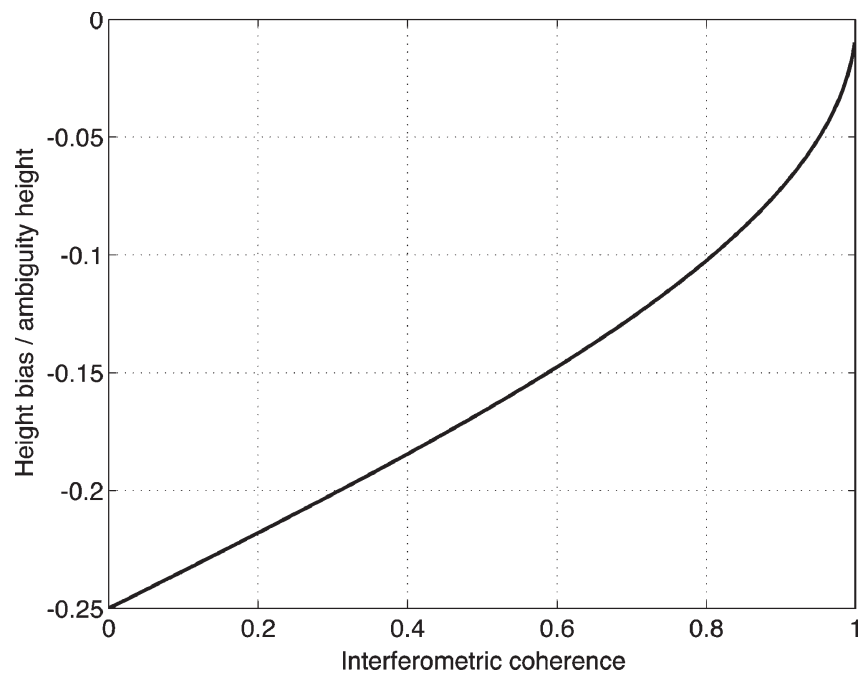

Fig. 3. Bias can be eliminated using the one-to-one relationship between the relative bias and the coherence magnitude.

normalization phase depends on the unknown surface elevation. However, the coherence phase is uniquely defined by the coherence magnitude, which in turn can be estimated from the InSAR data. Hence, using the coherent backscatter model, the elevation bias can be estimated from the coherence magnitude.

Fig. 1 illustrates geometrically the unique relationship between the coherence magnitude and the coherence phase, as a circle centered in origo has a unique intersection with the semicircle defined by the complex coherence. Mathematically, the coherence phase can be found from (9), which defines a one-to-one relationship between the coherence magnitude and the relative penetration depth $d_{2} / h_{a}$ from which the coherence phase can be computed

$$
\angle \gamma=-\operatorname{sgn}\left(h_{a}\right) \operatorname{Arctan}\left(\sqrt{|\gamma|^{-2}-1}\right) .
$$

The elevation bias is obtained by combining (12) and (15)

$$
\Delta_{h}=-\frac{\left|h_{a}\right|}{2 \pi} \operatorname{Arctan}\left(\sqrt{|\gamma|^{-2}-1}\right)
$$

The relative bias is plotted in Fig. 3 .

Hoen and Zebker [11] have related the one-way penetration length to the coherence magnitude. When rewritten in terms of the two-way penetration depth, their result is exactly what is found from (9)

$$
d_{2}=\frac{\left|h_{a}\right|}{2 \pi} \sqrt{|\gamma|^{-2}-1}
$$

Equations (16) and (17) are identical except for the sign and the Arctan. This is consistent with the fact that $|\gamma| \approx 1$ and $\operatorname{Arctan}(x) \approx x$ for $d_{2} / h_{a} \ll 1$.

In practice, bias mitigation based on (16) may be impaired by uncertainty on the correlation estimate, particularly when it is low, and by decorrelation due to noise, misregistration, temporal change, etc. Heavily vegetated areas, for instance, may exhibit a substantial temporal decorrelation, even with a

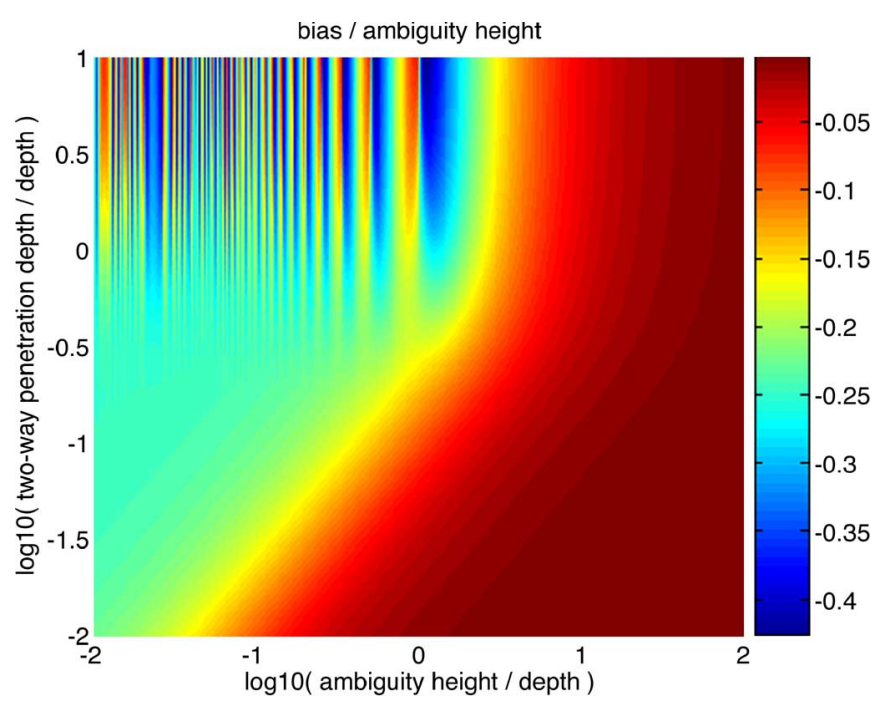

Fig. 4. Relative elevation bias $\left|\Delta_{h} / h_{a}\right|$. For penetration depths somewhat smaller than the volume depth, the relative elevation bias is virtually the same as for an infinitely deep volume.

short temporal baseline of one day [14]. Therefore, it is of interest to compute the sensitivity of the elevation bias to the coherence magnitude, as found from (16)

$$
\frac{\partial \Delta_{h}}{\partial|\gamma|}=\frac{\left|h_{a}\right|}{2 \pi \sqrt{1-|\gamma|^{2}}}
$$

The corresponding sensitivity for the penetration depth is found from (17)

$$
\frac{\partial d_{2}}{\partial|\gamma|}=-|\gamma|^{-2} \frac{\partial \Delta_{h}}{\partial|\gamma|}
$$

It is seen that, for coherence magnitudes somewhat lower than unity, the elevation bias is substantially less sensitive to the coherence magnitude errors than the penetration depth is. Hence, an inaccurate coherence magnitude estimate leads to a smaller inaccuracy on the bias estimate than on the penetration estimate, and so

- elevation bias estimation is more robust than penetrationdepth estimation.

\section{Finite-Volume Depth}

The findings in the preceding sections are based on a simple model of an infinitely deep volume. Since, in practice, all volumes have a finite depth, it is relevant to find the minimum depth, giving virtually the same results as an infinite depth. For volumes with a finite depth, the scattering properties of the bottom surface also become important. However, surface scattering is outside the scope of this paper.

According to (6) and (8), the complex coherence is a function of the three variables $D, d_{2}$, and $h_{a}$, but it can be rewritten in terms of only two variables, e.g., $d_{2} / D$ and $h_{a} / D$

$$
\gamma=\frac{D / d_{2}}{1-\exp \left(-D / d_{2}\right)} \cdot \frac{1-\exp \left(-D / d_{2}-j 2 \pi D / h_{a}\right)}{D / d_{2}+j 2 \pi D / h_{a}}
$$




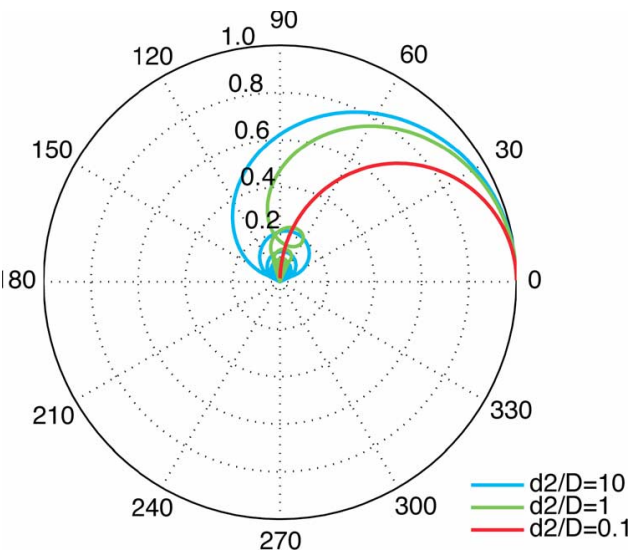

Fig. 5. InSAR coherence for three $d_{2} / D$ ratios plotted in the complex plane for variable $h_{a} / D$ ratio. A resonance phenomenon results when $d_{2} / D \geq 1$.

In addition, the relative elevation bias $\Delta_{h} / h_{a}$ that is found from (12) and (20) is a function of $d_{2} / D$ and $h_{a} / D$, as shown in Fig. 4. In the lower half of the figure, the relative bias is constant along the $45^{\circ}$ direction, i.e., it is constant for constant $d_{2} / h_{a}$ as it is for an infinitely deep volume, cf. Section III. The difference is smaller than $0.007 h_{a}$. On the other hand, in the upper half of the figure, where the $45^{\circ}$ invariability does not apply, the bias differs significantly from that of an infinitely deep volume. Hence,

- the volume depth can be considered infinite if it exceeds the penetration depth by a factor of two to five.

From (20) follows the well-known relationship [3]

$$
\gamma \rightarrow \exp \left(-j \pi D / h_{a}\right) \operatorname{sinc}\left(\pi D / h_{a}\right) \quad \text { for } \quad d_{2} / D \rightarrow \infty
$$

where $\operatorname{sinc}(x)=\sin (x) / x$. If additionally the ambiguity height is large compared with the volume depth, it follows from (21) that the bias is approximately half the volume depth, e.g., $\Delta_{h}=$ $-0.49 D$ in the upper right corner in Fig. 4

$$
\Delta_{h} \rightarrow-D / 2 \text { for } \quad d_{2} / D \rightarrow \infty,\left|h_{a}\right| / D \rightarrow \infty
$$

The oscillations in the upper left part of the figure is a resonance phenomenon occurring when the penetration depth is large compared with the volume depth, which in turn is large compared with the ambiguity height. The oscillations follow mathematically from (21), and they can be understood by considering the variation of the InSAR phasors with depth. When these phasors have almost the same length down through a layer of depth $h_{a}$, their mean sum is close to zero, since their angles are evenly distributed over 0 to $2 \pi$. Consequently, only a layer of depth $D$ modulo $h_{a}$ effectively contributes to the coherence. Fig. 5 shows, in polar coordinates, the coherence corresponding to three horizontal cuts in Fig. 4. It is seen that, when the penetration depth is reduced, the oscillations are damped, as the phasors no longer add up to zero when the phasor length varies significantly over a layer of depth of $h_{a}$.

\section{CONCLUSION}

Often, no clear distinction is made between the interferometric elevation bias and the penetration depth. Indeed, for an infinitely deep volume scatterer with negligible surface scattering, the absolute value of the bias almost equals the two-way power-penetration depth if the latter is much smaller than the ambiguity height. However, the bias cannot exceed one quarter of the ambiguity height, no matter the penetration depth. This implies that, unlike the interferometric height, the bias cannot wraparound.

The difference between bias and penetration depth may be quite significant in practice. When the penetration depth exceeds $10 \%$ of the ambiguity height, the interferometric bias is more than $10 \%$ smaller than the penetration depth, and when the penetration depth exceeds the ambiguity height, the bias is less than $10 \%$ from its maximum value.

The importance of distinguishing between bias and penetration depth can also be illustrated with the examples mentioned in the Introduction. The 11-m InSAR elevation bias measured in the percolation ice zone corresponds to a $12.8-\mathrm{m}$ two-way penetration depth. The measurement was made with the airborne EMISAR system, but assuming a mapping geometry like that of ENVISAT ASAR and a horizontal baseline of $200 \mathrm{~m}$, the same penetration length would result in an InSAR bias $64 \%$ smaller than the two-way penetration depth. The 120-m bias reported for cold exposed ice at L-band exceeds one quarter of the ambiguity height (if the TOPSAR ping-pong mode were used), so the model assumed in this paper is hardly applicable in this case. Most likely, the bedrock under the ice contributes significant surface scattering, thereby pulling the InSAR phasecenter downward.

Assuming the aforementioned satellite geometry, the 7-m penetration length in the tropical rain forest would result in an InSAR bias about 5\% smaller than the two-way penetration depth, while the corresponding number for the $22-\mathrm{m}$ penetration length in the boreal forest is $28 \%$. Finally, for the $2.5-\mathrm{m}$ dry-sand penetration length, the bias would merely be $0.12 \%$ smaller than the penetration depth.

The bias can be estimated from the magnitude of the interferometric coherence, thereby enabling bias mitigation. In this context, it should be noticed that the InSAR bias can be estimated more robustly than the penetration depth.

Although this paper has focused on infinitely deep volumes, the results are applicable to volumes deeper than two to five times the penetration depth, provided that the scattering from the top and bottom surfaces is negligible.

The highly idealized case defined in Section II does describe some real-world scenarios. For instance, the surface-to-volume scattering ratio for an ice sheet in the dry-snow zone is very small, particularly for large incidence angles. In addition, the top of a forest is not well defined and does not give rise to any surface scattering, and the success of the "random-volumeover-ground" model [4] used in Pol-InSAR mapping of forests suggests that a thick vegetation layer can often be considered uniform. On the other hand, the forest uniformity and, hence, the treetop elevation bias depends on the shape of the tree crowns [17]. In general, the validity of the assumptions made 
in this paper should be carefully assessed before applying the results.

Models describing a broader class of scenarios can also be based on the presented theory. For instance, InSAR elevationbias models have been developed for ice sheets with significant surface scattering and vertical nonuniformity [16]. In this case, a nonzero surface-to-volume scattering ratio reduces the bias while an increase of the extinction and scattering amplitude with depth enlarges the bias.

\section{APPENDIX}

The two-way propagation delay is the sum of the delay in air and the delay in volume

$$
\begin{aligned}
t_{d} & =\frac{2 H}{c \cos \theta_{a}}-\frac{2 n z}{c \cos \theta_{v}} \Leftrightarrow \\
z & =\frac{H \cos \theta_{v}}{n \cos \theta_{a}}-\frac{t_{d} c \cos \theta_{v}}{2 n}
\end{aligned} \Leftrightarrow
$$

where $c$ is the speed of the radar signal in air, $c / n$ is the speed in volume, and the minus sign in (A1) results from $z$ being negative in the volume according to the definition in Section II. The absolute interferometric phase $\phi$ is proportional to the projection of the baseline onto the line-of-sight direction

$$
\phi=\frac{2 \pi p}{\lambda} B \cdot \sin \left(\theta_{a}-\alpha\right)
$$

where $\alpha$ is the baseline angle with respect to the horizontal, and $B$ is the baseline length, which is assumed to be much smaller than the slant range. For fixed $t_{d}$, the sensitivity of the interferometric phase to the depth of the scattering center can be found as

$$
\begin{aligned}
\frac{\partial \phi}{\partial z} & =\frac{\partial \phi}{\partial \sin \theta_{a}}\left(\frac{\partial z}{\partial \sin \theta_{a}}\right)^{-1} \\
& =\frac{2 \pi p B_{\perp}}{\lambda \cos \theta_{a}}\left(\frac{H \tan \theta_{a} \cos \theta_{v}}{n \cos ^{2} \theta_{a}}-\frac{\tan \theta_{v}}{n^{2}}\left(\frac{H}{\cos \theta_{a}}-\frac{c t_{d}}{2}\right)\right)^{-1} \\
& =\frac{2 \pi p B_{\perp}}{\lambda \cos \theta_{a}}\left(H \tan \theta_{a} \frac{\cos \theta_{v}}{n \cos ^{2} \theta_{a}}+\frac{z \tan \theta_{v}}{n \cos \theta_{v}}\right)^{-1} .
\end{aligned}
$$

Here, Snell's law, $\sin \theta_{a}=n \sin \theta_{v}$, has been applied, and $t_{d}$ has been eliminated using (A1). Assuming that $z \ll H$

$$
\frac{\partial \phi}{\partial z} \approx \frac{2 \pi p B_{\perp}}{\lambda \cos \theta_{a}}\left(H \tan \theta_{a} \frac{\cos \theta_{v}}{n \cos ^{2} \theta_{a}}\right)^{-1}
$$

Applying Snell's law once again and introducing the vertical wavenumber from (4) lead to

$$
k_{z}=\frac{2 \pi p B_{\perp}}{\lambda H \tan \theta_{a}} \frac{n^{2} \cos \theta_{a}}{\sqrt{n^{2}-\sin ^{2} \theta_{a}}} .
$$

\section{REFERENCES}

[1] A. Reigber and A. Moraira, "First demonstration of airborne SAR tomography using multi-baseline L-band data," IEEE Trans. Geosci. Remote Sens., vol. 38, no. 5, pp. 2142-2152, Sep. 2000.

[2] S. R. Cloude and K. P. Papathanassiou, "Polarimetric SAR interferometry," IEEE Trans. Geosci. Remote Sens., vol. 36, no. 5, pp. 1551-1565, Sep. 1998.

[3] S. R. Cloude and K. P. Papathanassiou, "Three-stage inversion process for polarimetric SAR interferometry," Proc. Inst. Electr. Eng.-Radar Sonar Navig., vol. 150, no. 3, pp. 125-134, Jun. 2003.

[4] K. P. Papathanassiou and S. R. Cloude, "Single-baseline polarimetric SAR interferometry," IEEE Trans. Geosci. Remote Sens., vol. 39, no. 11, pp. 2352-2363, Nov. 2001.

[5] E. Rignot, K. Echelmeyer, and W. Krabill, "Penetration depth of interferometric synthetic-aperture radar signals in snow and ice," Geophys. Res. Lett., vol. 28, no. 18, pp. 3501-3504, 2001.

[6] J. Dall, S. N. Madsen, K. Keller, and R. Forsberg, "Topography and penetration of the Greenland ice sheet measured with airborne SAR interferometry," Geophys. Res. Lett., vol. 28, no. 9, pp. 1703-1706, May 2001.

[7] C. Varekamp and D. H. Hoekman, "High-resolution InSAR image simulation for forest canopies," IEEE Trans. Geosci. Remote Sens., vol. 40, no. 7, pp. 1648-1655, Jul. 2002.

[8] R. N. Treuhaft, S. N. Madsen, M. Moghaddam, and J. J. van Zyl, "Vegetation characteristics and underlying topography from interferometric radar," Radio Sci., vol. 31, no. 6, pp. 1449-1485, Nov./Dec. 1996.

[9] K. K. Williams and R. Greeley, "Radar attenuation by sand: Laboratory measurements of radar transmission," IEEE Trans. Geosci. Remote Sens., vol. 39, no. 11, pp. 2521-2526, Sep. 2001.

[10] J. F. McCauley, G. G. Schaber, C. S. Breed, M. J. Grolier, C. V. Haynes, B. Issawi, C. Elachi, and R. Blom, "Subsurface valleys and geoarcheology of the Eastern Sahara revealed by Shuttle radar," Science, vol. 218, no. 4576, pp. 1004-1020, Dec. 1982.

[11] E. W. Hoen and H. A. Zebker, "Penetration depths inferred from interferometric volume decorrelation observed over the Greenland ice sheet," IEEE Trans. Geosci. Remote Sens., vol. 38, no. 6, pp. 2571-2583, Sep. 2000.

[12] R. N. Treuhaft and P. R. Siqueira, "Vertical structure of vegetated land surfaces from interferometric and polarimetric radar," Radio Sci., vol. 35, no. 1 , pp. 141-177, 2000

[13] F. Gatelli, A. M. Guarnieri, F. Parizzi, P. Pasquali, C. Prati, and F. Rocca, "The wavenumber shift in SAR interferometry," IEEE Trans. Geosci. Remote Sens., vol. 32, no. 4, pp. 855-865, Jul. 1994.

[14] P. A. Rosen, S. Hensley, I. R. Joughin, F. K. Li, S. N. Madsen, E. Rodríguez, and R. M. Goldstein, "Synthetic aperture radar interferometry," Proc. IEEE, vol. 88, no. 3, pp. 333-382, Mar. 2000.

[15] M. Santoro, J. Askne, and P. B. G. Dammert, "Tree height influence on ERS interferometric phase in boreal forest," IEEE Trans. Geosci. Remote Sens., vol. 43, no. 2, pp. 207-217, Feb. 2005.

[16] J. Dall, K. P. Papathanassiou, and H. Skriver, "Polarimetric SAR interferometry applied to land ice: Modeling," in Proc. EUSAR, Ulm, Germany, May 2004, pp. 247-250.

[17] I. Izzawati, E. D. Wallington, and I. H. Woodhouse, "Forest height retrieval from commercial X-band SAR products," IEEE Trans. Geosci. Remote Sens., vol. 44, no. 4, pp. 863-870, Apr. 2006.

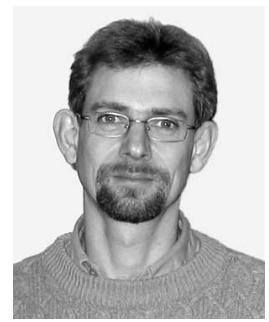

Jørgen Dall (M'07) received the M.Sc. degree in electrical engineering and the Ph.D. degree from the Technical University of Denmark, Kongens Lyngby, Denmark, in 1984 and 1989, respectively.

In the 1990s, he worked with the Danish airborne SAR system, EMISAR. He led the development of dedicated onboard and offline processors, and he was responsible for the data processing. From 1996 to 2001, he also organized the EMISAR dataacquisition campaigns and served as Mission Manager. Since 1991, he has been an Associate Professor at the Technical University of Denmark, Kongens Lyngby, Denmark. Currently, he is leading the development of an airborne polarimetric ice sounding radar for the European Space Agency. His research includes design of advanced radar altimeters for planetary missions, development of SAR signal-processing techniques, polarimetric SAR interferometry, polarimetric and interferometric SAR calibrations, and SAR applications within geology and ice mapping. 